\title{
Innovation, implementation and organisational tension
}

Please visit the QSHC website (http://www.qshc.com) for links to these articles - many to full text.

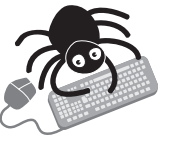

The eight papers below each relate to different dimensions of the same problem: how organisational innovation can ride the tensions that proposals for change inevitably generate.

The papers can be divided into three areas:

(1) efforts still being developed to improve patient and public involvement in health services;

(2) more established attempts to diffuse innovation through structural change, a performance assessment system, the use of clinical guidelines, and through exploiting organisational culture and shaping individual identity; and

(3) a systematic review of the diffusion of innovation and a catalogue of the complexities involved and tensions that different approaches generate; some interesting suggestions are made about how to engage with these.

\section{Improving patient and public involvement}

Some innovations strive to fundamentally change relationships between patients and health professionals, health services and their users.

Citizens' juries to set local health needs? Involving the public has become so important that, according to Kashefi and Mort, it has become a "bureaucratic preoccupation for every health agency in the UK". The problem is that health agencies tend to approach consultation in a tick box fashion rather than genuinely gather views to improve local services. The authors testify this routine: "we are repeatedly invited to submit proposals for consultation projects knowing what is being sought is the public view at a competitive price rather than critical engagement and identification of complexities".

This paper explores "an innovative approach for local participation" through the development of "a grounded citizens' jury", which was promoted by the IPPR on the basis of research into similar models in the US and Germany. In south west Burnley (a town in Northern England) 12 local people were recruited to a citizens' jury to set questions, invite evidence and deliberate on what they felt would improve the health and well being of people living in the area. The question chosen was simply: "What would improve the health and well-being of residents in south west Burnley?" The researchers acted as facilitators to the jury who invited their own witnesses and were encouraged to reach a verdict (make recommendations) after deliberation. This was transcribed and passed to a local group of managers and professionals.

The aim of the researchers was to encourage the jury to join this group, bringing its 80 recommendations and help to change local health services. But while the jurors had been initially confident about their ideas, "this belief was challenged once they interacted with lengthy bureaucratic processes and professionals who had their own knowledge and asserted their own expertise". Jurors came to "feel sidelined and ignored and lost the motivation to remain involved". The group could not sustain a relationship with local health planners.

The authors argue that "local consultation strategies need to be underpinned by sustained development work with communities so as to build general capacity to participate".

A Kashefi E, Mort M. Grounded citizens' juries: a tool for health activism? Health Expectations 2004;7:290-302.

How does trust affect patient preferences for participation in decision making? The second paper, also from Health Expectations, from a team of Canadian researchers begins by asking the question: "Does trust in physicians aid or hinder patient autonomy?"

606 patients responded to a survey. They were shown vignettes and asked: "Who should diagnose, determine treatment options, identify the risks and benefits for each option, and how likely these risks and benefits are likely to happen?" They could answer (1) the doctor alone, (2) mostly the doctor, (3) both equally, (4) mostly me or (5) me alone. They were also asked to indicate their level of trust in doctors and this was compared with the stated preference for participation in decision making to see if there were any correlations.

People with low trust of doctors tend to prefer autonomy in decision making and a loss of public trust would indicate a shifting social preference to take decisions autonomously. Just over $6 \%$ professed "blind trust" in their physician, 36.1\% had high trust, $48.6 \%$ moderate trust, and $9 \%$ said they had "low trust". Levels of education, gender and age were all significant variables on levels of trust.

Although blind trust is not common, it was seen more frequently among female than male respondents, among those with less education, and those over 65 years of age. In contrast, it was never found among those with post-secondary education or those under 35 years of age. Fewer than $3 \%$ preferred an autonomous role and almost all preferred to make decisions more or less equally with the doctor. The authors state that: "Although most of our respondents rejected a purely passive role, they also showed little desire to take full charge".

Trust is a prerequisite for shared decision making and, in supporting the majority's preference for shared decision making, interventions must take care not to undermine this.

$\Delta$ Kraetschmer N, Sharpe N, Urowitz S, et al. How does trust affect patient preferences for participation in decision-making? Health Expectations 2004;7:317-26.

What mode of decision making do doctors generally follow? Another paper from Canada, again from Health Expectations, looked at the extent to which Ontario breast cancer specialists practise shared decision making with their patients, their comfort level with this approach, and their perceptions of the things that block and aid its implementation.

One of the problems in assessing shared decision making is that there is little consensus on the meaning of this term, making it difficult to know whether it is being achieved in practice. A similar ambiguity is present is many new policy initiatives. To help understand how doctors understood shared decision making, the researchers asked a group of oncologists and surgeons to identify which of the following approaches they understood as shared and which resembled their general mode of working with patients. The examples of different modes of decision making are paraphrased below:

(1) The doctor decides on a suitable treatment and presents this, giving information about the treatment, including risks and benefits.

(2) The doctor presents the available treatment options, information about the risks and benefits of each are discussed, and the doctor recommends a treatment.

(3) The doctor presents the available treatment options, information about the risks and benefits of each are given and discussed, and the doctor asks the patient to make the decision.

(4) The doctor presents available treatment options, shares information about risks and benefits, invites questions and asks about preferences, lifestyle and important issues through which a decision is reached.

No labels were attached to the four clinical examples and doctors were asked about their relative comfort with each of the approaches. The majority said they were comfortable with example 4; half were comfortable with example 2; $35 \%$ of oncologists and $42 \%$ of surgeons were comfortable with example 3 ; and none were 
comfortable with example 1. $56 \%$ of oncologists and $69 \%$ of surgeons cited example 4 as their usual approach; $27 \%$ of oncologists and $17 \%$ of surgeons said their approach was mostly like example 2; few of either specialty indicated that their approach was usually like example 3 and even fewer like example 1.

It is interesting that, while doctors say they are comfortable with shared decision making, they are not always able to put these aspirations into practice. Barriers to shared decision making included: lack of time to spend with patients; patient anxiety about their condition that prevents open discussion; patients' lack of information or high levels of misinformation; and, in a significant number of cases, an unwillingness or inability to participate.

The authors consider that "it is the physician's role to actively elicit patient preferences in this regard, rather than to assume what the patient is thinking". They conclude that "more research is needed to identify contextual, physician, patient and interaction factors that will facilitate shared decision making in the medical encounter and help both parties create an environment conducive to implementing this approach to the extent desired".

The paper is a reminder that shared decision making might vary from person to person. Greater clarity is needed around the roles of doctor and patient in shared decision making so that each can be sure about the role the other wants them to play. Too little is written about the interpersonal relationship that underpins shared decision making as well as the mode of interaction that will best afford it.

$\Delta$ Charles C, Gafni A, Whelan T. Self-reported use of shared decision-making among breast cancer specialists and perceived barriers and facilitators to implementing this approach. Health Expectations 2004;7:338-48.

Diffusing innovation $>$ The next four papers look at attempts to diffuse innovation in organisations.

Clinicians' view of the clinical directorate organisational model A paper from Australia looks at structural change and its success in making health professionals and management a more coherent group. The paper examines assertions made by proponents and critics of clinical directorate (CD) structures in hospitals.

Seventy eight doctors, 89 nurses and 60 allied health professionals working in two large Australian hospitals that had implemented CDs 3 years previously completed a survey in which they were asked to indicate, on a Likert scale ranging from "strongly agree" to "strongly disagree", whether they believed a suggested outcome had occurred as a result of the move to a CD structure. Overall, more were negative than positive. Staff most commonly concurred with the statements that the introduction of CDs has: led to an increase in organisational politics $(58 \%)$, improved accountability $(48 \%)$, been responsible for dumping hard decisions on staff $(39 \%)$. Only a relatively small number felt it had improved patient care $(26 \%)$. What is most interesting is the level and nature of disagreement across the groups. While $60 \%$ of nurses believed CDs have increased accountability, the same proportion of doctors believed it had not. While over half the doctors thought the new structure "dumped hard decisions on staff", almost $64 \%$ of nurses did not.

The most statistically significant result from the research was the contrast found between doctors' belief (84.4\%) that they did not have much say in how things were run and others professionals' perceptions (nurses $52.8 \%$ and allied health $51.7 \%$ ) that doctors did have too much power.

A clear finding is that "CDs are perhaps necessary but not sufficient in themselves to overcome entrenched barriers to multidisciplinary teams. It may be that cultural change at the microbehavioural level-in terms of the situated practices of clinicians in wards, units and departments (i.e. at the sub directorate level)-is what will count in the end; although professional tribes will doubtless remain a powerful force of countervailing pressure".

The paper calls on policy to concentrate on "change at the work group level if only because change at the CD level does not appear on these results to have delivered to stakeholders what proponents believed it would: professional collaboration on care across traditional professional divides". In conclusion, the authors call for "a rethink of what can be expected from structural reforms in organisations".
A Braithwaite J, Westbrook M. Rethinking clinical organisational structures: an attitude survey of doctors, nurses and allied health staff in clinical directorates. $J$ Health Serv Res Policy 2005; 10:10-17.

Influence of performance assessment systems on organisational culture An alternative approach is to use performance assessment to catalyse organisational change. A study undertaken in England explores some of the impacts of star performance ratings" $-a$ system in which the achievements of healthcare organisations against a range of measures are brought together to produce a single rating $(0,1,2$ or 3 stars). Ratings were published for the first time in September 2001.

The paper shows a few problems in the configuration of the star rating system across the board. While the star ratings were viewed by some managers as useful in that they concentrated minds on certain targets, "there was a general view that the star ratings as presently constituted did not represent a rounded or balanced scorecard of their own organisation's performance and a widespread belief that the information used to calculate the ratings was often incomplete and inaccurate" ${ }^{\prime \prime}$. In addition, the ratings were also sometimes reported to have inadvertently induced a range of unintended and dysfunctional consequences by focusing management attention on one area at the exclusion of another, leading to "tunnel vision" and ultimately "a distortion of clinical priorities". The system led to friction in internal relationships as managers sought to direct activities that were relevant to their ratings and led to "various degrees of bullying, intimidation and harassment of staff in the apparently under-performing trusts".

The authors argue that external assessment can act as "a lever to influence staff behaviour" as managers are keen to perform well and will focus their energies on what is being measured. This is why it is so important to get the measures right. They highlight a number of important gaps in what is known about the use of performance assessment to lever improvement and suggest the following questions to help explore these further.

- To what extent do global measures such as star ratings capture a valid and reliable assessment of overall performance?

- What is the most appropriate unit of analysis for rating the performance of health services?

- What is the most appropriate time period to be covered by performance ratings?

- How can performance assessments be presented in ways that both capture attention and garner credibility?

- How can organisations integrate internal quality improvement activities with external assessments of performance?

- What dysfunctional consequences are being stimulated within organisations, how important are they, and how can they be mitigated?

The authors conclude that "the many dysfunctional consequences of publishing star ratings indicate a need for a re-examination of performance management policies".

$\Delta$ Mannion R, Davies $H$, Marshall M. Impact of star performance ratings in English acute hospital trusts. J Health Serv Res Policy 2005;10:18-24.

Adoption of national guidelines by local organisations - A paper from England observes the responses of three primary care practices to the first four National Service Frameworks (NSFs). The focus of the study is on how local GP practices approach these clinical and service guidelines.

Cross analysis of the three practices found a similar pattern in their responses even though one had been actively involved in clinical guidelines. This suggests that these findings were "not a product of more general attitudes to the overall agenda of change". People in all practices spoke positively about the idea of clinical guidelines. They offered practical help in managing patients. However, when they were asked about actual NSFs, "attitudes were more negative, and concentrated on the failure of NSFs to offer practical help"

People seemed to think it was about doing "a better job for everyone in the universe. But fine, Right. How are you going to do that? Give me the details?"

The proportion of staff that had read one or more of the first four NSFs was very low. The most widely read was the guidance on CHD-over half had read the summary and $10 \%$ the whole document. Only $10 \%$ had read the summary NSF on elderly people 
and nobody had read the full document. There was no evidence of one person reading the document and cascading their knowledge.

One doctor summed up the problems they felt with pace of change and heavy workload: "If anyone thinks that things arrive here and somebody with the ability to look at it properly has the time to look at it and then spread it as useful information that everyone else thinks sensibly about, they've got another think coming"

The author says that clear and simple guidelines for the care of asthma from the British Thoracic Society had been adopted. NSFs, by contrast, were dismissed with others as "too complicated" and resource consuming. NSFs have the capacity to increase workload further for already overworked doctors. She notes that "as nurses spend their time with these patients, work that has traditionally been done by practice nurses will have to be done by someone elsemost likely the doctors". "Time spent on developing call and recall systems .... is time that cannot be spent on other things". While not arguing that working to implement the NSF is wrong, the author feels that "it could be said to be inappropriate in the specific circumstances of this practice, as they struggled to provide appointments for the patients who presented".

"The fact that GPs in the three practices with a track record of adopting clinical guidelines did not specifically address centrally imposed NSFs can more easily be explained by the failure of these weighty documents to make the job easier than by invoking profession-wide concerns relating to autonomy and control over working practices."

The paper concludes by calling for research to better understand "the sense that individuals make of their working lives" which may, when set alongside other knowledge, "contribute to a deeper understanding of the complex responses to centrally-imposed change".

$\Delta$ Checkland K. National Service Frameworks and UK general practitioners: street-level bureaucrats at work? Sociol Health Illn 2004;26:951-75.

Creating a new organisational identity The final paper in this section explores the notion of "empowerment" and more cultural approaches to stimulate innovation within organisational settings.

One way of seeing empowerment is in relation to "entrepreneurial thinking with the aim of encouraging associated qualities such as initiative, self-reliance and the ability to accept responsibility for oneself and one's actions". More critical commentators have interpreted the focus on empowerment as a management tool for "exercising control over employees and their identities".

McDonald explores the validity of this critical view through the experience of an English primary care organisation which is newly formed and is setting out by seeking to "empower its senior staff'. The primary care trust (PCT) was formed in April 2001 from two primary care groups and one community health services trust. " $A$ study commissioned from a third party by the PCT chief executive found low morale, widespread dissatisfaction with channels of communication and distrust of senior management of the PCT. Respondents reported feeling vulnerable and complained of a lack of organisational identity." The external consultants reported that "many employees felt a sense of belonging in relation to the existing services they provided and their immediate working relationships".

The views expressed in the document were seen as dangerous as they set out an alternative view of "resistant discourse". "The reporting of such views in a quasi-official publication created the possibility that, once circulated throughout the organisations, such findings would lend legitimacy to the discourse of opposition". Copies of the report were destroyed and the PCT set about trying to forge a singular identity.

The PCT chief executive extended an invitation to all staff to nominate people in the PCT who were "key influencers"; these members of staff were then invited to participate in an "Investing in Excellence" programme. At the programme they were split into groups and brought together as a group for 5 days over 12 months.

Some found the course very useful. One person said: "Before the programme I would just react to something and sometimes quite inappropriately as well maybe lose my temper and slamming and banging ... I'm not where I want to be but I'm getting there" researcher's critical eye acknowledges that this is "suggestive of a self in transition" but sees the course as encroaching into personality training. "The implication is that outward displays of emotion are quite inappropriate behaviour for the ethical self to which she aspires".
Another person recognised the way they are changing. "I've been a little bit unhappy because I've heard comments from friends that a couple of mangers have commented that 'Jackie has changed' but, yes, Jackie has changed as a consequence of the programme ... to me that was the whole point of the programme. You do change as a persona professionally and personally ... I should have changed otherwise the programme would not have been a success. I realise that my comments and opinions are as valid as anybody else's in the organisation and your manager or the boss may not necessarily always be right and l've got a right to have an opinion. I'll challenge things but the point is it's not liked if you challenge something."

In this regard, the approach of the PCT to empower individuals may backfire. Talking about the PCT identity and vision had served to alienate some people. Although it is assumed that "organisational goals are internally consistent and compatible", the course showed that the objectives of two employees working for the same PCT can conflict. "The Deputy Director of Public Health was dismayed by the fact that others in the PCT choose to prioritise waiting lists rather than tackling health inequalities". "The Deputy Director of Teaching and Learning was disappointed by the way in which the PCT 'top level' had failed to appreciate its importance as a core function underpinning the PCT's work".

"Government proclamations that staff are now in the driving seat ignores the fact that empowered staff members may try to steer the vehicle in many directions at the same time, thereby creating tensions amongst employees. This tension appears to manifest itself in disappointment with the attitude of others and a willingness to redefine what is important in the organisations, rather than in attempts to modify one's own notion of what is ethical behaviour."

A McDonald R. Individual identity and organisational control: empowerment and modernisation in a primary care trust. Sociol Health Illn 2004;26:925-50.

\section{The complexities of diffusing innovation}

The final paper summarises an extensive overview of the issues surrounding the diffusion of innovation in health and service settings. It was commissioned by the UK Department of Health and carried out between October 2002 and December 2004. It offers some insight into the thinking around how quality and safety initiatives can be taken forward and how better to negotiate the tensions described in previous papers.

The review looked across a wide array of literature to examine the question: "How can we spread and sustain innovations in health service delivery and organization?" The authors became aware that the health literature itself contained too few studies. Over time, they purposefully undertook a meta-narrative review - which is a focus on the unfolding "story line" of research in a particular research tradition.

The paper reviews approaches to diffusion from a wide range of academic disciplines. "Because different researchers in different traditions generally conceptualized their topic differently, used different language and metaphors for diffusion, dissemination, and implementation; asked different questions; privileged different methods; and used different criteria to judge 'quality' and 'success', we used narrative rather than statistical synthesis techniques".

The research team found 13 research areas that were relevant:

- Rural sociology: the study of society and the relationship between its members, especially the influence of social structures and norms.

- Medical sociology: as above for the medical society.

- Communications studies: psychological studies of human communication.

- Marketing: economic and psychological studies of the production, distribution and consumption of goods and services. Traditionally, these research areas developed discretely but shared "erroneous assumptions" that (1) the only relevant unit of analysis is the individual innovation, (2) an innovation is necessarily better than what went before, (3) patterns of adoption reflect fixed personality traits, and (4) that findings are transferable to new settings.

New disciplines have shed light on new ways to understand innovation. These new areas include: 
- Developmental studies: studies of the adoption, adaptation, and use of technology.

- Health promotion: strategies and practices to improve the health and wellbeing of populations.

- Evidence based medicine: clinical epidemiology.

- Structural determinants: how structure influences function.

- Organisational studies: development and impact of culture, groups and influence.

- Inter-organisational studies: social and virtual networks, strategy.

- Knowledge utilisations: study of how individuals and teams acquire, structure, synthesise and share knowledge.

- Narrative studies: studies of stories (told in organisations).

- Complexity studies: studies of how individuals, groups, and organisations emerge and involve.

Approaches to innovation can be crudely mapped on a spectrum from "let it happen" to "make it happen" and variously involve managerial, technical, social, and natural or emergent mechanisms. They range between a view of change as unpredictable and emergent and one that sees it as scientifically orderly and manageable. Between them is a more political perspective seeing change as negotiated, influenced and enabled.

Many innovations go wrong and some of the reasons need to be explored, although they are often complicated. Too often, models of diffusion and innovation are seen as more linear than research shows them to be. "Even so called evidence based innovations undergo a lengthy period of negotiation among potential adopters".

"People are not passive recipients of innovations. Rather (and to a greater or lesser extent in different persons), they seek innovations, experiment with them, evaluate them, find (or fail to find) meaning in them, develop feelings (positive or negative) about them, challenge them, gain experience with them, modify them to fit particular tasks, and try to improve or redesign them-often through dialogue with other users. This diverse list of actions and feelings highlights the complex nature of adoption as a process and contrasts markedly with the widely cited early/later adopter categories that have been extensively misapplied."

Although there are over 1000 papers relevant to this area, very few acknowledge the "important issue of internal politics". Another striking finding of this extensive review was "the tiny proportion of empirical studies that acknowledged, let alone explicitly set out to study, the complexities of spreading and sustaining innovation".
The authors suggest future research on health service innovations should be:

- theory driven-exploring hypothesised links;

- process rather than package orientated;

- ecological-recognise dynamic links in complex organisations;

- addressed using common definitions, measures and tools to enable valid comparisons across studies;

- collaborative and coordinated-projects should focus on questions across multiple programs rather than isolate small stories;

- multi-disciplinary and multi-method-emphasising different interpretations;

- meticulously detailed; and

- participatory-involving practitioners as partners in the research process.

$\Delta$ Greenhalgh T, Robert G, MacFarlane F, et al. Diffusion of innovations in service organizations: systematic review and recommendations. Milbank $Q$ 2004;82:581-629.

\section{Conclusions}

Research continually reminds us that innovation and the implementation of initiatives are extraordinarily complex. No organisational initiative can plan for every detail or manifestation of the tensions that will be inextricably linked to a change initiative. But knowing that tension is inevitable means they should be acknowledged and help us to debate.

Sometimes tensions are to do with a mismatch between aims and too few opportunities to state aims and explore tensions between them. At other times, tensions result from there being no clear link between professional and managerial views of structures and processes.

What seems important, however, is to establish local processes that link clinical teams in forums through which different perspectives and ideas can be exchanged and discussed. Those concerned with the spread of innovation need to push for the creation of process, structures, and information systems to develop and sustain capacity for clinical teams to access, assess, discuss, and evaluate new ideas and information.

T Smith

Senior Policy Analyst, Health Policy and Economic Research Unit, British Medical Association, London WC1H 9JR, UK; tsmith@bma.org.uk

Register now!

10th European Forum on Quality Improvement in Health Care 13-15 April 2005, ExCel Conference Centre, London For further information on how to register please go to: http://www.quality.bmipg.com 